\title{
SIMULATION OF INERTIAL NAVIGATION SYSTEM ERRORS AT AERIAL PHOTOGRAPHY FROM UAV
}

\author{
R. Shults \\ Kyiv National University of Construction and Architecture, Faculty for Geoinformation Systems and Territory Management, \\ Povitroflotskyi Avenue, 31 Kyiv, 03037, Ukraine, shultsrv@gmail.com
}

Commission V, WG V/7

KEY WORDS: Unmanned aerial vehicle, Aerial photography, Inertial navigation system (INS), Accelerometer systematic shift, Gyroscope systematic offset, Position accuracy

\begin{abstract}
:
The problem of accuracy determination of the UAV position using INS at aerial photography can be resolved in two different ways: modelling of measurement errors or in-field calibration for INS. The paper presents the results of INS errors research by mathematical modelling. In paper were considered the following steps: developing of INS computer model; carrying out INS simulation; using reference data without errors, estimation of errors and their influence on maps creation accuracy by UAV data. It must be remembered that the values of orientation angles and the coordinates of the projection centre may change abruptly due to the influence of the atmosphere (different air density, wind, etc.). Therefore, the mathematical model of the INS was constructed taking into account the use of different models of wind gusts. For simulation were used typical characteristics of micro electromechanical (MEMS) INS and parameters of standard atmosphere. According to the simulation established domination of INS systematic errors that accumulate during the execution of photographing and require compensation mechanism, especially for orientation angles. MEMS INS have a high level of noise at the system input. Thanks to the developed model, we are able to investigate separately the impact of noise in the absence of systematic errors. According to the research was found that on the interval of observations in 5 seconds the impact of random and systematic component is almost the same. The developed model of INS errors studies was implemented in Matlab software environment and without problems can be improved and enhanced with new blocks.
\end{abstract}

\section{INTRODUCTION}

The last 25 years in the world, we have seen a stable increase in requirements for geospatial data. These requirements differ but generally this is desire to improve the accuracy and detail of data collection and at the same time increasing the speed and reducing cost. In the traditional version with the aim of mapping and GIS projects data were collected using traditional methods of terrestrial survey or aerial photography. Terrestrial technologies are complex and energy-intensive and not very suitable for fast and detailed data collection and updating. On the other hand, the traditional aerial photography with long distance from the camera to the object does not allow completely display all its characteristics, and the results are highly dependent on weather conditions. Both technologies are expensive and therefore not very suitable for frequent data updates. Today, very popular is method of data collection using GNSS (global navigation satellite system). However, this method is actually a continuation of traditional terrestrial survey because it requires direct determination of each point, although however slightly reducing the cost of work and time spent on data collection.

An alternative to existing methods of data collection is the use of technology, which in complex use different navigation technologies and remote sensing (Bosak, 2014). These technologies include aerial photography using unmanned aerial vehicles (UAV). The main advantage of this technology is the simultaneous reduction of cost and time for data collection. In comparison with aerial photography, UAV camera equipment is much simpler, the surveying distances are smaller and the efficiency is much higher (Colomina et al. 2014). Of course, aerial photography from the UAVs has its drawbacks, such as low accuracy at high altitudes. However, in many projects UAV benefits are more significant in comparison with disadvantages. Aerial photography from the UAVs provides the accuracy that requires the most of the topographical work. However, achieving the required level of accuracy is challenging. The main problems in obtaining accurate data are concern to navigation facilities as UAV navigation unit is a complex system with many sensors that have completely different nature of information. UAV navigation equipment may include GNSS, barometric sensor, magnetic compass, tilt sensor, gyroscopes system, accelerometers systems, INS. The best solution for aerial photography using UAVs is navigation unit GNSS/INS. Due to the high cost and bulkiness of accurate inertial navigation systems for UAV are used miniature electromechanical navigation systems (MEMS). These INSs have undeniable advantages from the point of cost and dimensions. However, the accuracy of inertial systems wishes to better. Accelerometers and gyroscopes that are part of the miniature navigation systems have low measurement accuracy. Since determination of the position and orientation in space, using INS based on double integration of the measured accelerations and angular velocities, even minor errors at the beginning of integration growing very quickly over time. For INS errors correction are using two approaches:

- The study of a particular model of INS and construction of mathematical dependencies, which describe system errors.

- INS correction at specified intervals, using GNSS data.

The first approach is suitable for high precision, tactical-grade INS for which errors are stable and do not change over time. INS of this type cannot be used for UAV due to high cost and 
huge weight. For low-cost INS rational is use of GNSS for periodic correction (Abdel-Hamid, 2005; Grejner-Brzezinska, et al. 2004) rather than calibration (Artese et al. 2008), due to large and unstable errors. The choice of the interval by which INS correction is performed depends on how quickly accumulating errors and changing accuracy of INS in intervals between corrections. Finally, from this depends the accuracy with which the position of the UAV and the position and orientation of aerial equipment will be determined (Rehak et al., 2014). So, important is the task of INS errors simulation and based on such research, setting INS correction interval using GNSS. Such researches will allow choosing INS with proper characteristics. Therefore, the main idea of this paper is practical research of low-cost INS accuracy by mathematical simulation results. Based on these results we tried to establish the influence of INS position on accuracy of topographic maps creation.

First of all we have to establish the mathematical model of INS.

\section{INS MODEL}

The main coordinate systems, which are using in inertial navigation, are inertial system (i-system), earth centered system (e-system), local horizontal navigation system (n-system), body coordinate system (b-system). The core description and determination of these systems you can find in (Biezad , 1999; Salytcheva, 2004).

The orientation of UAV can be determined by three angles ( $\varphi$ roll, $\theta$ - pitch, $\psi$ - heading), which need for connection between vectors in b-system and n-system at the same point in space.

We chose the INS model in which the angles and coordinates get in n-system. In such case, we introduce a short description of this model according to (Zang, 2003).

The equations of position and orientation calculation in $n-$ system have the next form:

$$
\left[\begin{array}{c}
\dot{\mathbf{r}}^{n} \\
\dot{\mathbf{v}}^{n} \\
\dot{\mathbf{R}}_{b}^{n}
\end{array}\right]=\left[\begin{array}{c}
\mathbf{D}^{-1} \mathbf{v}^{n} \\
\mathbf{R}_{b}^{n} \mathbf{a}^{b}-\left(2 \mathbf{\Omega}_{i e}^{n}+\mathbf{\Omega}_{e n}^{n}\right) \times \mathbf{v}^{n}+\boldsymbol{\gamma}^{n} \\
\mathbf{R}_{b}^{n}\left(\boldsymbol{\omega}_{i b}^{b}+\mathbf{\Omega}_{i n}^{b}\right)
\end{array}\right],
$$

where $\omega_{i e}=$ Earth rotation velocity

$\mathbf{a}^{b}=$ measured accelerations vector in b-system

$\boldsymbol{\omega}_{i b}^{b}=$ measured angular velocities vector in b-system

$\left(2 \mathbf{\Omega}_{i e}^{n}+\mathbf{\Omega}_{e n}^{n}\right) \times \mathbf{v}^{n}=$ vector corrections for influence of Coriolis and centrifugal accelerations

$\boldsymbol{\gamma}^{n}=$ vector of acceleration of normal gravity

$\boldsymbol{\Omega}_{i e}^{e}=\left[\begin{array}{c}0 \\ 0 \\ \omega_{e}\end{array}\right] ; \boldsymbol{\Omega}_{i e}^{n}=\mathbf{R}_{e}^{n} \boldsymbol{\Omega}_{i e}^{e}=\left[\begin{array}{c}\omega_{i e} \mathrm{c} B \\ 0 \\ -\omega_{i e} \mathrm{~s} B\end{array}\right]$,

$\mathbf{D}=\left[\begin{array}{ccc}\frac{1}{M+H} & 0 & 0 \\ 0 & \frac{1}{(N+H) \mathrm{c} B} & 0 \\ 0 & 0 & -1\end{array}\right]$.

Rotation from b-system to n-system is describing by next matrix:

$$
\mathbf{R}_{b}^{n}=\left[\begin{array}{ccc}
\mathrm{c} \psi \mathrm{c} \theta & -\mathrm{s} \psi \mathrm{c} \varphi+\mathrm{c} \psi \mathrm{s} \theta \mathrm{s} \varphi & \mathrm{s} \psi \mathrm{s} \varphi+\mathrm{c} \psi \mathrm{s} \theta \mathrm{c} \varphi \\
\mathrm{s} \psi \mathrm{c} \theta & \mathrm{c} \psi \mathrm{c} \varphi+\mathrm{s} \psi \mathrm{s} \theta \mathrm{s} \varphi & -\mathrm{c} \psi \mathrm{s} \varphi+\mathrm{s} \psi \mathrm{s} \theta \mathrm{c} \varphi \\
-\mathrm{s} \theta & \mathrm{c} \theta \mathrm{s} \varphi & \mathrm{c} \theta \mathrm{c} \varphi
\end{array}\right],
$$

Rotation from n-system to e-system can be implemented through the transition velocity:

$$
\boldsymbol{\Omega}_{e n}^{n}=\left[\begin{array}{c}
\frac{v_{E}}{N+H} \\
-\frac{v_{N}}{M+H} \\
-\frac{v_{E} \operatorname{tg} B}{N+H}
\end{array}\right]=\left[\begin{array}{l}
\omega_{e n_{x}}^{n} \\
\omega_{e n_{y}}^{n} \\
\omega_{e n_{z}}^{n}
\end{array}\right],
$$

where $B, L, H=$ geodetic coordinates

$v_{E}, v_{N}=$ east and north components of velocity

$M, N=$ main radius of ellipsoid

Total rotation from e-system to n-system is describing by next matrix:

$$
\boldsymbol{\Omega}_{e n}^{n}=\left[\begin{array}{ccc}
-\mathrm{s} B \mathrm{c} L & -\mathrm{s} B \mathrm{~s} L & \mathrm{c} B \\
-\mathrm{s} L & \mathrm{c} L & 0 \\
-\mathrm{c} B \mathrm{c} L & -\mathrm{c} B \mathrm{~s} L & -\mathrm{s} B
\end{array}\right]
$$

In expressions, superscript indicates the coordinate system in which parameters are presented, and the bottom shows the original system of coordinates. For angular velocities, lower index indicates in respect of which two coordinate systems occurs rotation.

Computational model of INS, which is implemented in the nsystem shown in Figure 1. Calculations in this scheme do with equations (1).

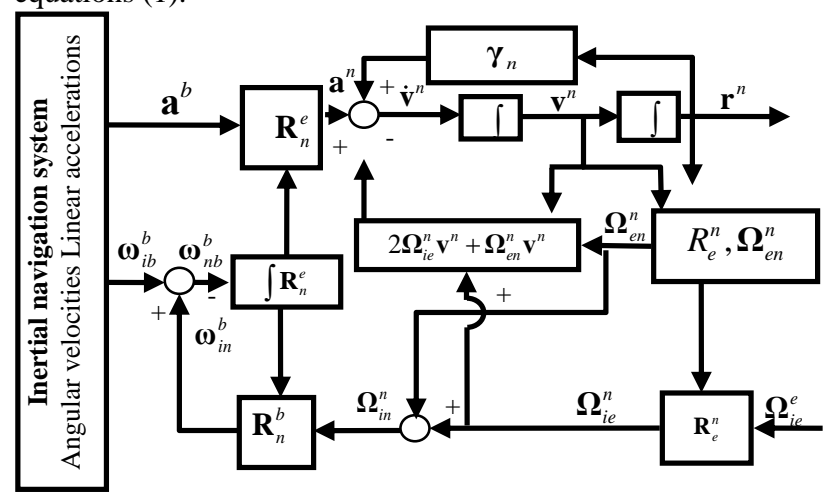

Figure 1. INS mechanisation (Zang, 2005)

Now we defined INS mathematical model. The next step is to choose correct model for describing INS errors.

\section{INS ERRORS MODEL}

The main measuring devices of INS are gyroscopes and accelerometers. These devices have special errors, which depend on structure. That is why for follow simulation is important to choose correct INS errors model. For such model, we have to choose measurement model for both gyroscopes and accelerometers.

The equation of measured accelerations in matrix form will (Park, 2004):

$$
\mathbf{a}^{b}=\mathbf{a}+\delta \mathbf{a}+\mathbf{s}_{a} \mathbf{a}+\mathbf{N}_{a} \mathbf{a}+\boldsymbol{\gamma}+\delta \boldsymbol{\gamma}+\boldsymbol{\varepsilon}_{a},
$$

where $\mathbf{a}=$ vector of clear accelerations

$\delta \mathbf{a}=$ vector of accelerometers systematic bias

$\mathbf{s}_{a}=$ matrix of scale coefficients

$\mathbf{N}_{a}=$ matrix of angular accelerometers misalignments 
$\gamma=$ vector acceleration of normal gravity

$\delta \gamma=$ vector of acceleration of normal gravity variations

$\boldsymbol{\varepsilon}_{a}=$ vector of accelerations measurements noise

Also, equation (5) in expanded form will:

$$
\begin{aligned}
& {\left[\begin{array}{l}
a_{X}^{b} \\
a_{Y}^{b} \\
a_{Z}^{b}
\end{array}\right]=\left[\begin{array}{l}
a_{X} \\
a_{Y} \\
a_{Z}
\end{array}\right]+\left[\begin{array}{l}
\delta a_{X} \\
\delta a_{Y} \\
\delta a_{Z}
\end{array}\right]+\left[\begin{array}{ccc}
s_{X} & \Delta \psi & -\Delta \theta \\
-\Delta \psi & s_{Y} & \Delta \varphi \\
\Delta \theta & -\Delta \varphi & s_{Z}
\end{array}\right]\left[\begin{array}{l}
a_{X} \\
a_{Y} \\
a_{Z}
\end{array}\right]+} \\
& {\left[\begin{array}{c}
-\gamma \sin \theta \\
\gamma \cos \theta \sin \varphi \\
\gamma \cos \theta \cos \varphi
\end{array}\right]+\left[\begin{array}{c}
\delta \gamma_{X} \\
\delta \gamma_{Y} \\
\delta \gamma_{Z}
\end{array}\right]+\left[\begin{array}{c}
\varepsilon_{a_{X}} \\
\varepsilon_{a_{Y}} \\
\varepsilon_{a_{Z}}
\end{array}\right] .}
\end{aligned}
$$

The equation of measured angular velocities in matrix form will:

$$
\boldsymbol{\omega}_{i b}^{b}=\boldsymbol{\omega}+\delta \boldsymbol{\omega}+\mathbf{s}_{\omega} \boldsymbol{\omega}+\mathbf{N}_{\omega} \boldsymbol{\omega}+\boldsymbol{\varepsilon}_{\omega},
$$

where $\boldsymbol{\omega}=$ vector of clear angular velocities

$\delta \omega=$ vector of gyroscopes systematic bias

$\mathbf{s}_{\omega}=$ matrix of scale coefficients

$\mathbf{N}_{\omega}=$ matrix of angular gyroscopes misalignments

$\boldsymbol{\varepsilon}_{\omega}=$ vector of gyroscopes measurements noise

Also, equation (6) in expanded form will:

$$
\left[\begin{array}{c}
\omega_{X}^{b} \\
\omega_{Y}^{b} \\
\omega_{Z}^{b}
\end{array}\right]=\left[\begin{array}{l}
\omega_{X} \\
\omega_{Y} \\
\omega_{Z}
\end{array}\right]+\left[\begin{array}{l}
\delta \omega_{X} \\
\delta \omega_{Y} \\
\delta \omega_{Z}
\end{array}\right]+\left[\begin{array}{ccc}
s_{X} & \Delta \psi & -\Delta \theta \\
-\Delta \psi & s_{Y} & \Delta \varphi \\
\Delta \theta & -\Delta \varphi & s_{Z}
\end{array}\right]\left[\begin{array}{l}
\omega_{X} \\
\omega_{Y} \\
\omega_{Z}
\end{array}\right]+\left[\begin{array}{c}
\varepsilon_{X} \\
\varepsilon_{Y} \\
\varepsilon_{Z}
\end{array}\right] .
$$

In the most cases, the random part is presented as a sum of white noise and additional components.

$$
\varepsilon=\varepsilon_{W}+\varepsilon_{c}+\varepsilon_{r}+\varepsilon_{q}+\varepsilon_{t},
$$

where $\varepsilon_{W}=$ white noise

$$
\begin{aligned}
& \varepsilon_{c}=\text { correlation noise } \\
& \varepsilon_{r}=\text { random shift } \\
& \varepsilon_{q}=\text { quantization noise } \\
& \varepsilon_{t}=\text { trembling noise }
\end{aligned}
$$

The total errors model of random and systematic errors of accelerometers and gyroscopes for simulation are possible to present by equations (5) and (6) using devices specifications.

\section{INITIAL DATA AND MATLAB SIMULINK MODEL FOR SIMULATION}

For our simulation, we need two types of initial data: technical specifications of INS and simulated clear accelerations and angular velocities. In order to construct errors model we chose follow errors values (Goodall et al. 2012; Barret, 2014), which presented in table below.

\begin{tabular}{|l|l|}
\hline Error & Value \\
\hline Accelerometer bias $\delta a$ & $100 \mu \mathrm{g}, 250 \mu \mathrm{g}, 500 \mu \mathrm{g}$ \\
\hline Axis non-orthogonality $\Delta$ & $60^{\prime \prime}$ \\
\hline Gyroscope bias $\delta \omega$ & $1,5^{\circ} / \mathrm{hr}, 2,5^{\circ} / \mathrm{hr} 5^{\circ} / \mathrm{hr}$ \\
\hline Accelerometers noise, RMS & $5 \mathrm{mg} / \mathrm{hr} / \sqrt{\mathrm{Hz}}$ \\
\hline Gyroscopes noise, RMS & $0.5^{\circ} / \mathrm{hr} / \sqrt{\mathrm{Hz}}$ \\
\hline
\end{tabular}

Table 1. INS errors

According to large value of biases, the scale coefficients of accelerometers and gyroscopes were neglected.

In the case of measured accelerations and angular velocities were chosen three types of trajectories: straight spatial line, classical surveying trajectory for one route (is using for linear objects) and complicated curve, which has a form of "eight" (often is using for INS calibration). All these trajectories presented below.

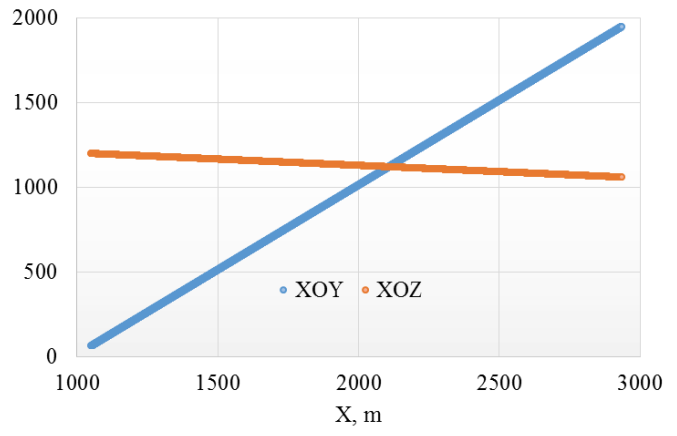

Figure 2. Projection of spatial line on planes XOY and XOZ

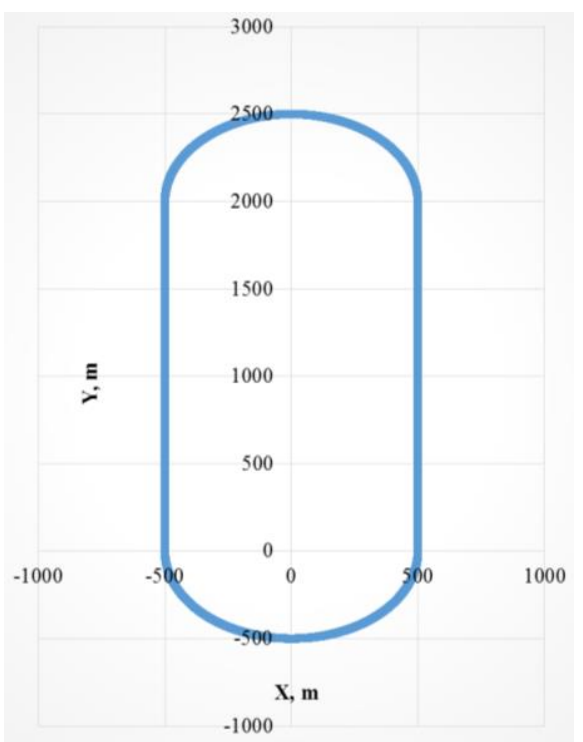

Figure 3. Closed route along linear object

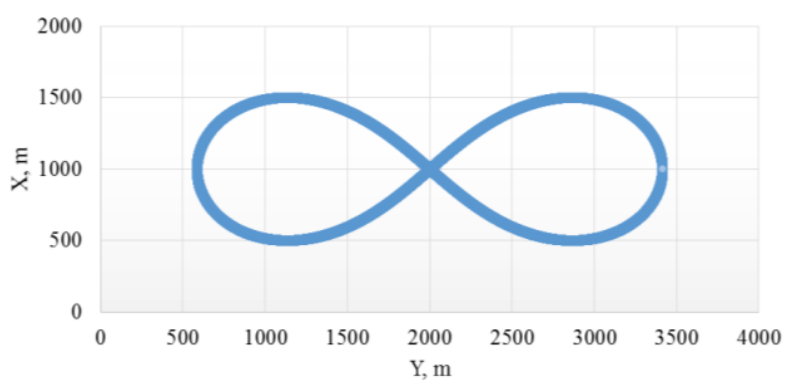

Figure 4. Complicated curve

The movement of UAV has taken uniform, with a constant speed of $80 \mathrm{~km} / \mathrm{h}$. Using equations (1) the Matlab Simulink model was created (Figure 5). The study was set up m-file, which also ran simulations of two INS models, with the influence of errors and without. During the simulation automatically were formed at the same time the differences between two position coordinates. The coordinate's differences were obtained for all three axes X, Y, Z. There were performed 27 launches of Matlab Simulink model. Nine launches were made with the assumption that no error of gyroscopes. The accelerometers errors consistently taken the values $100 \mu \mathrm{g}, 250$ $\mu \mathrm{g}, 500 \mu \mathrm{g}$. The next nine launches INS were made with the assumption that no error of accelerometers. The gyroscopes errors consistently take the values of $1.5^{\circ} / \mathrm{h}, 2.5^{\circ} / \mathrm{h}, 5^{\circ} / \mathrm{h}$. One of the aims of this research was to determine the effect separately 
gyroscopes and accelerometers. The final option was executed and gyroscopes. nine studies that included the presence of errors accelerometers

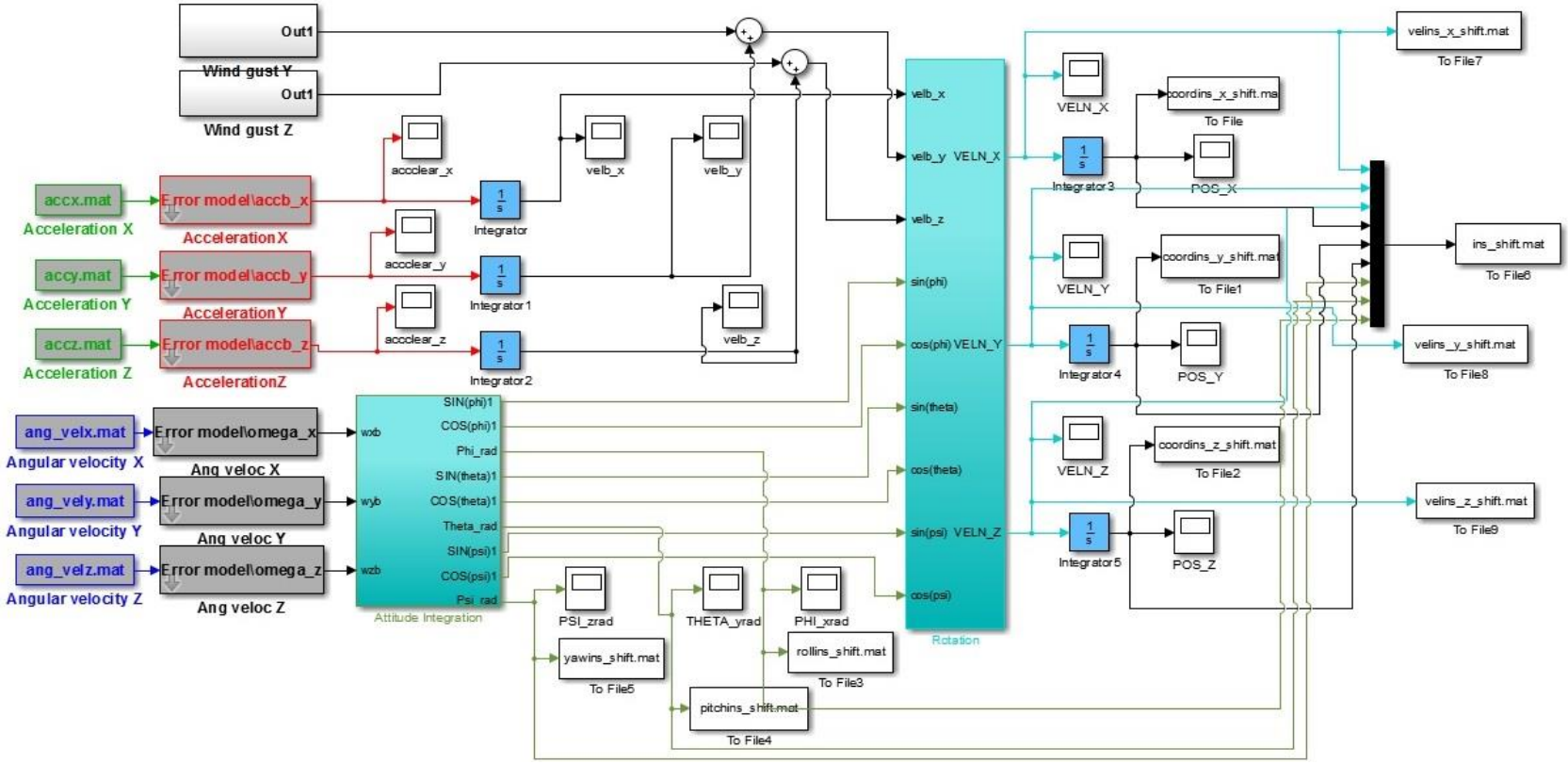

Figure 5. Matlab Simulink model of INS

In this case were formed three errors models: first model (1) $100 \mu \mathrm{g} / 1,5^{\circ} / \mathrm{hr}$; second model (2) - $250 \mu \mathrm{g} / 2,5^{\circ} / \mathrm{hr}$; third model (3) $-500 \mu \mathrm{g} / 5^{\circ} / \mathrm{hr}$. Other INS errors were left without changing in all launches. The total time of simulation was $2 \mathrm{~min}$. Sampling frequency was $100 \mathrm{~Hz}$.

\section{INS SIMULATION RESULTS}

Here we present the simulation results. The most comfortable is presenting these results in graphic form. Due to lack of paper volume, we are presenting just a little part of results. The simpler interpretation of errors accumulation can be made for trajectory of straight spatial line. Below presented the results of errors accumulation along $\mathrm{X}$ axis.

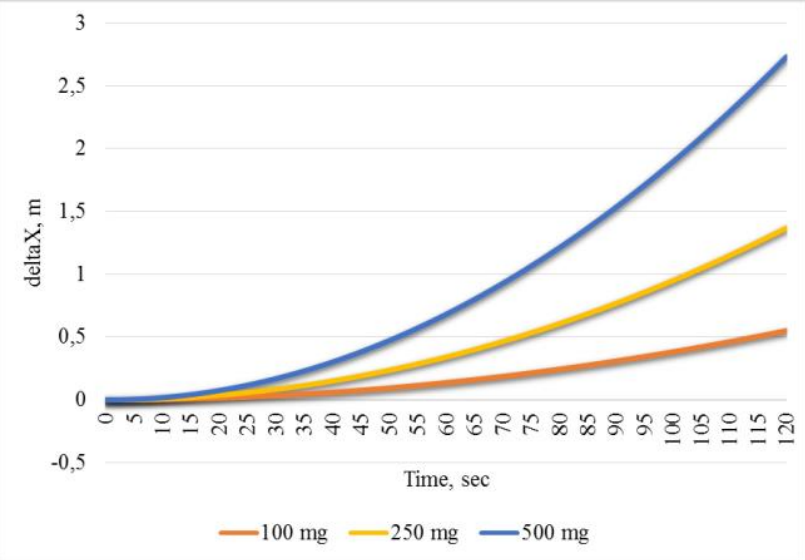

Figure 6. Accelerometers errors accumulation along $X$ axis

As we can see, in the modern MEMS INSs the level of errors is too high. For distance $2 \mathrm{~km}$, the displacement along $\mathrm{X}$ axis add up to $1 / 8$ from distance and grows by exponent.

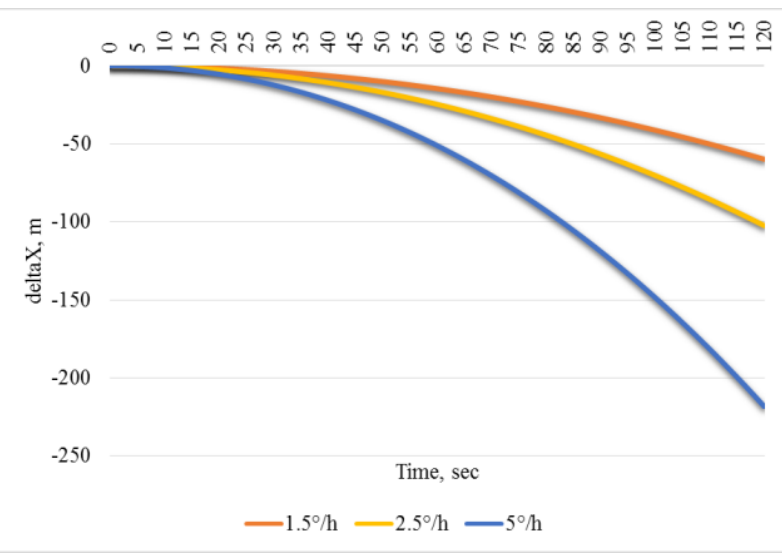

Figure 7. Gyroscopes errors accumulation along $\mathrm{X}$ axis

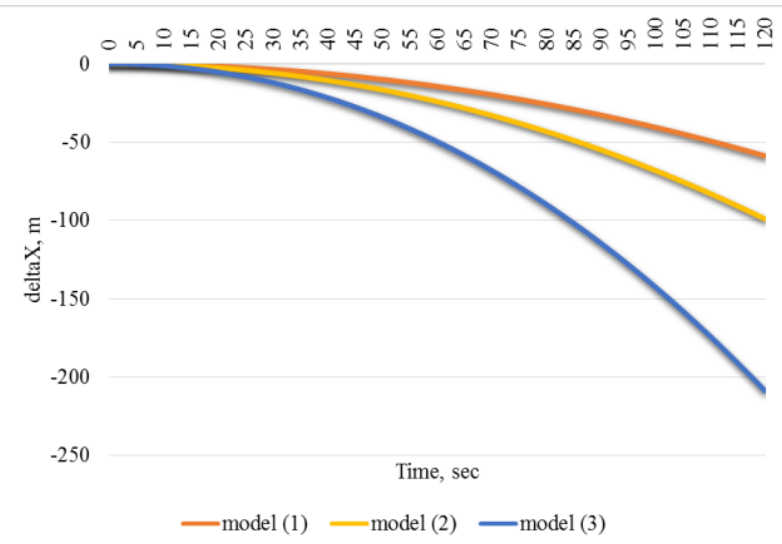

Figure 8. Total errors accumulation along $\mathrm{X}$ axis

So, it is interesting to define in what time period the INS errors will have accepted values. For this, we presented the graphics of errors accumulation along $\mathrm{Y}$ axis for 2 seconds time interval. As in the previous case, we are presenting displacements along $\mathrm{Y}$ axis for three variants. 


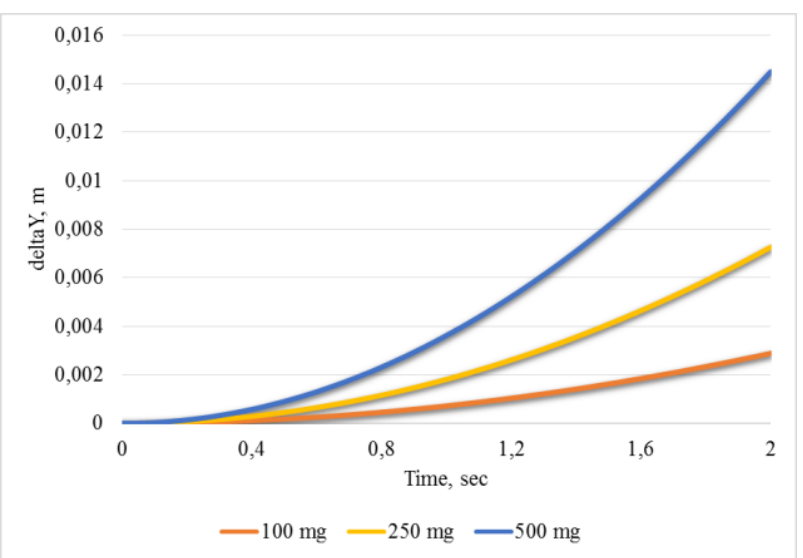

Figure 9. Accelerometers errors accumulation along $\mathrm{Y}$ axis

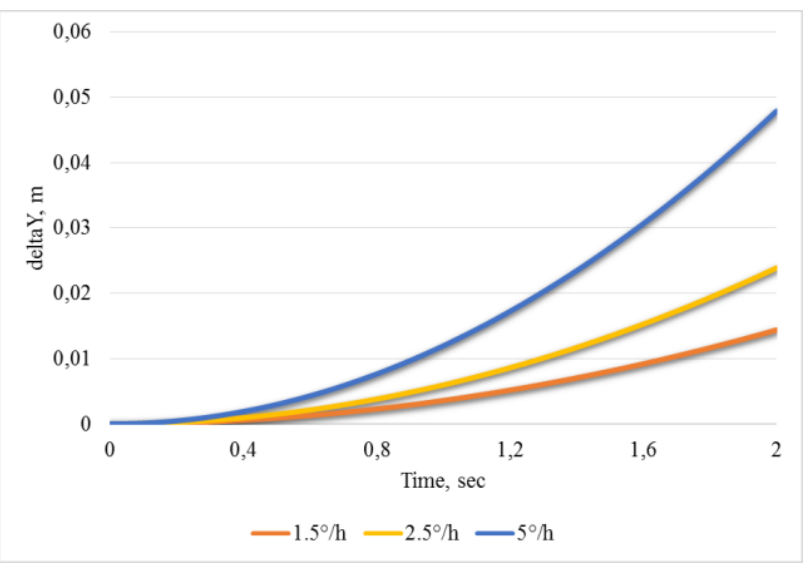

Figure 10. Gyroscopes errors accumulation along $\mathrm{Y}$ axis

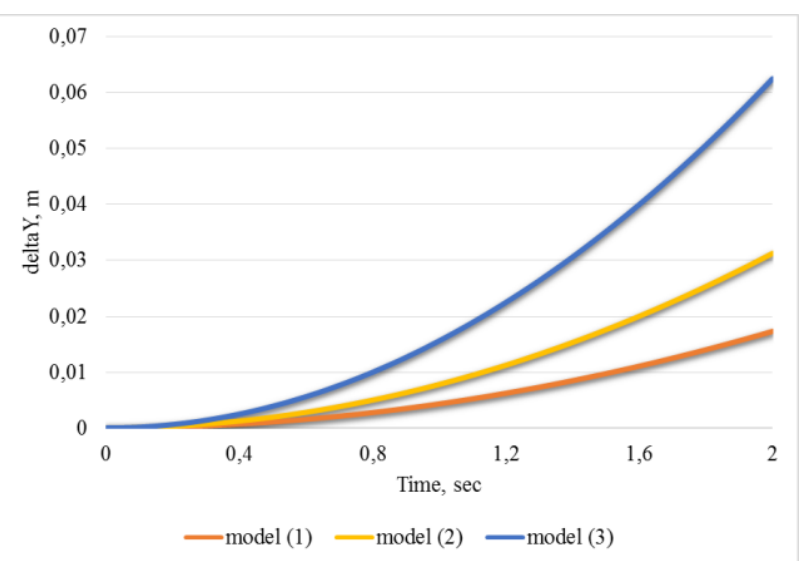

Figure 11. Total errors accumulation along $\mathrm{Y}$ axis

From Figures 9-11, we can conclude that total errors of INS have normal values. These results are very useful as allow finding and establishing correct time interval for INS correction by GNSS.

Another one useful property of constructed Matlab model is possibility to research how the wind gusts can influence on accuracy of position determination. Below presented such simulations for model (1).

These results are quite interesting and need more dipper analysis. Anyway, we can conclude that such atmosphere phenomenon as wind gusts have significant influence on INS accuracy.

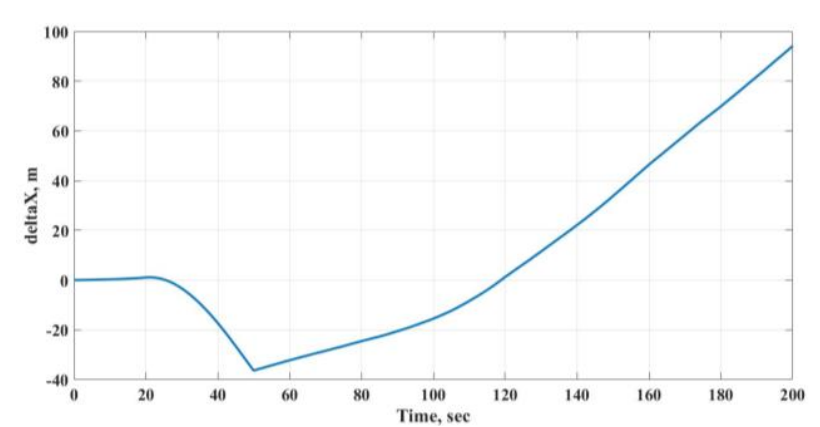

Figure 12. Changing of INS error along $\mathrm{X}$ axis, including wind gust

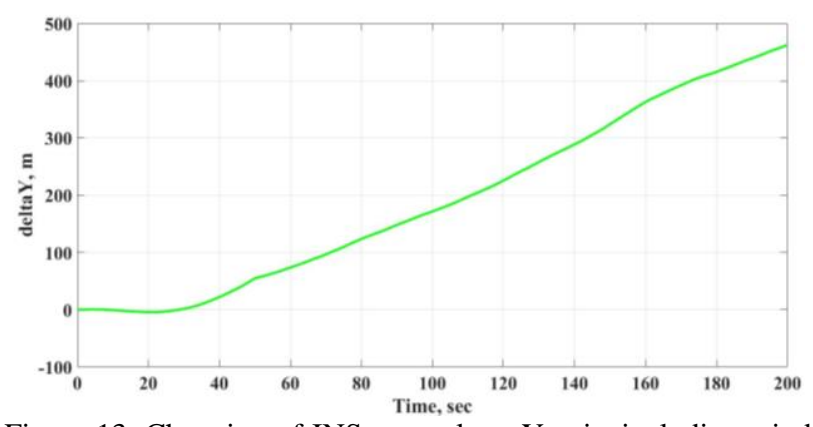

Figure 13. Changing of INS error along Y axis, including wind gust

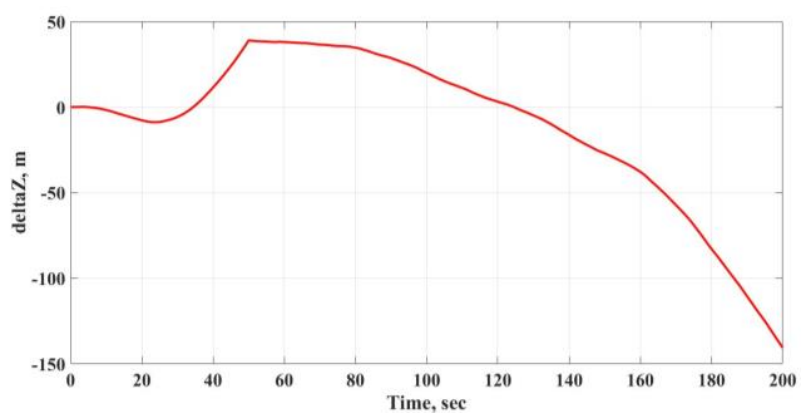

Figure 14. Changing of INS error along $\mathrm{Z}$ axis, including wind gust

After this, the last step is results analysis

\section{RESULTS ANALYSIS}

To perform the analysis of the results we have to find out how the errors of INS affecting on topographical maps accuracy.

In surveying assumed that if a source of error does not exceed $1 / 5$ of the total value of the error, its influence is negligible. In this case, we are considering the expected errors in scale of aerial photographs. We can write:

$$
\frac{\delta_{X_{I N S}}}{m} \leq \frac{1}{5} \delta_{x} ; \frac{\delta_{Y_{I N S}}}{m} \leq \frac{1}{5} \delta_{y} ; \frac{p \delta_{Z_{I N S}}}{H} \leq \frac{1}{5} \delta_{p}
$$

where $\delta_{X_{I N S}}, \delta_{Y_{I N S}}, \delta_{Z_{I N S}}=$ coordinates determination errors (from INS simulation)

$\delta_{x}, \delta_{y}, \delta_{p}=\delta_{z}=$ errors of coordinates and parallaxes measurements on image

$$
m \text { = image scale } 4000 \text { (for focus } 50 \mathrm{~mm} \text { and }
$$
surveying height $200 \mathrm{~m}$ )

$p$ = parallax, $5 \mathrm{~mm}$ on image (for $80 \%$ overlap)

If we accept a scales of topographic maps 1:500, 1:1000, 1:2000, 1:5000, the error in determining the position will: 


$$
\delta_{X}, \delta_{Y}=\frac{0.4 M}{\sqrt{2}},
$$

where $M$ = map scale.

For height we will have

$$
\delta_{Z}=\frac{1}{3} h,
$$

where $h=$ vertical interval, which will take for these scales 0.5 $\mathrm{m}, 1.0 \mathrm{~m}, 1.0 \mathrm{~m}, 2.0 \mathrm{~m}$.

Using the above value by expressions (9-10) we calculate the acceptable points errors. Next, by the expressions (8) we calculate the acceptable points errors on the image and points errors on image due to errors INS for time interval of 2 seconds (model 3). From the acceptable errors we move to values which can be neglected and compare these values with INS errors.

Again, we will use simulation data for straight spatial line. The calculation results are given in tables.

\begin{tabular}{|c|c|c|c|}
\hline Scale & $\begin{array}{c}\text { Error on map } \\
\delta_{X}, \mathrm{~m}\end{array}$ & $\begin{array}{c}\text { Error on image } \delta_{x}, \\
\mathrm{~mm}\left(0.2 \delta_{x} \mathrm{~mm}\right)\end{array}$ & $\begin{array}{c}\text { INS error } \delta_{X_{I N S}} \text { on } \\
\text { image, } \mathrm{mm \kappa}\end{array}$ \\
\hline 500 & 0,14 & $0,035(0,007)$ & 0,013 \\
\hline 1000 & 0,28 & $0,070(0,014)$ & 0,013 \\
\hline 2000 & 0,57 & $0,140(0,028)$ & 0,013 \\
\hline 5000 & 1,43 & $0,350(0,070)$ & 0,013 \\
\hline
\end{tabular}

Table 2. Comparative analysis of calculated and acceptable INS errors (axis X)

\begin{tabular}{|c|c|c|c|}
\hline Scale & $\begin{array}{c}\text { Error on } \\
\text { map } \delta_{Y}, \mathrm{~m}\end{array}$ & $\begin{array}{c}\text { Error on image } \delta_{y}, \\
\mathrm{~mm}\left(0.2 \delta_{y} \mathrm{~mm}\right)\end{array}$ & $\begin{array}{c}\text { INS error } \delta_{Y_{I N S}} \text { on } \\
\text { image, } \mathrm{mm \kappa}\end{array}$ \\
\hline 500 & 0,14 & $0,035(0,007)$ & 0,016 \\
\hline 1000 & 0,28 & $0,070(0,014)$ & 0,016 \\
\hline 2000 & 0,57 & $0,140(0,028)$ & 0,016 \\
\hline 5000 & 1,43 & $0,350(0,070)$ & 0,016 \\
\hline
\end{tabular}

Table 3. Comparative analysis of calculated and acceptable INS errors (axis Y)

\begin{tabular}{|c|c|c|c|}
\hline Scale & $\begin{array}{c}\text { Error on } \\
\text { map } \delta_{Z}, \mathrm{~m}\end{array}$ & $\begin{array}{c}\text { Error on image } \delta_{z}, \\
\mathrm{~mm}\left(0.2 \delta_{z} \mathrm{~mm}\right)\end{array}$ & $\begin{array}{c}\text { INS error } \delta_{Z_{I N S}} \text { on } \\
\text { image, } \mathrm{mm \kappa}\end{array}$ \\
\hline 500 & $0,17(0,5)$ & $0,004(0,0008)$ & 0,0013 \\
\hline 1000 & $0,33(1,0)$ & $0,008(0,0016)$ & 0,0013 \\
\hline 2000 & $0,33(1,0)$ & $0,008(0,0016)$ & 0,0013 \\
\hline 5000 & $0,67(2,0)$ & $0,017(0,0032)$ & 0,0013 \\
\hline
\end{tabular}

Table 4. Comparative analysis of calculated and acceptable INS errors (axis Z)

If we assume that GNSS works with frequency $1 \mathrm{~Hz}$ and can determine the coordinates with precision $0,02-0,03 \mathrm{~m}$ in any axis, then INS allows to determine with the necessary accuracy the position of the UAV in the intervals between the GNSSmeasurements. On the observation interval of 2 seconds INS can be used when creating topographical maps of scale 1: 1000 .

\section{CONCLUSIONS}

In presented paper was constructed and investigated mathematical model of INS errors. The research established the dominance of systematic errors of INS that accumulate during the performance of aerial photography from UAV and require compensation mechanism, especially for orientation angles. It was established that low cost INS have another characteristic feature. This is the high level of noise at the system input. Thanks to the model developed by us, we are able to examine separately the impact of noise in the absence of systematic errors.

The accuracy of INS was simulated for different operating time. For the 5 seconds time interval was established that random and systematic impact component is almost the same. Therefore, when performing coordinates correction by GNSS, the assessment of systematic component impact can be done only on time intervals from 10 seconds. At the same time the rate of accumulation of errors in angular orientation is slower. In this case can be recommended to use the gyroscopes errors model.

The influence of wind gusts on INS coordinates were carried out. These results need future investigations. One of the way in which it can be done, it is using vehicle dynamic model as it was made in paper (Khaghani et al., 2016).

At the end of a paper was given methodic of assessment INS errors affecting on topographical maps accuracy. Therefore we can assess the influence of INS errors and choose proper navigation equipment.

\section{REFERENCES}

Abdel-Hamid, W., 2005. Accuracy Enhancement of Integrated MEMS-IMU/GPS Systems for Land Vehicular Navigation Applications. A Thesis for the Degree Doctor of Philosophy. Calgary.

Artese, G., Trecroci A., 2008. Calibration of a low cost MEMS INS sensor for an integrated navigation system. In: The International Archives of the Photogrammetry, Remote Sensing and Spatial Information Sciences, Beijing, China, Vol. XXXVII, Part B5, pp. 877-882.

Barret, J.M., 2014. Analyzing and modeling low-cost MEMS IMUs for use in an inertial navigation system. A Thesis for the Degree of Master of Science. Worcester.

Biezad, D.J., 1999. Integrated Navigation and Guidance Systems. American Institute of Aeronautics and Astronautics, Reston, 242 p.

Bosak, K., 2014. Secrets of UAV photomapping http://s3.amazonaws.com/DroneMapper_US/documentation/pte ryx-mapping-secrets.pdf.

Colomina, I., Molina, P., 2014. Unmanned aerial systems for photogrammetry and remote sensing: A review. ISPRS Journal of Photogrammetry and Remote Sensing, Vol. 92, pp. 79-97. dx.doi.org/10.1015/j.isprsjprs.2014.02.013.

Goodall, C., Carmichael, S., El-Sheimy, N., Scannell, B., 2012. INS Face Off MEMS versus FOGs. InsideGNSS, JULY/AUGUST, pp. 48-55.

Grejner-Brzezinska, D.A., Toth, C.K., 2004. High-Accuracy Direct Aerial Platform Orientation with Tightly Coupled GPS/INS System. Ohio Department of Transportation, Office of Aerial Engineering, Federal Highway Administration.

Khaghani, M., Skaloud, J., 2016. Application of vehicle dynamic modeling in UAVs for precise determination of exterior prientation. In: The International Archives of the 
Photogrammetry, Remote Sensing and Spatial Information Sciences, Prague, Czech Republic, Vol. XLI-B3, pp. 827-831.

Park, M., 2004. Error Analysis and Stochastic Modeling of MEMS based Inertial Sensors for Land Vehicle Navigation Applications. A Thesis for the Degree Doctor of Philosophy. Calgary

Rehak, M., Mabillard, R., Skaloud, J., 2014. A Micro Aerial Vehicle with Precise Position and Attitude Sensors. PFG Photogrammetrie, Fernerkundung, Geoinformation, Issue 4, pp. 239-251, DOI: $10.1127 / 1432-8364 / 2014 / 0240$

Salytcheva, A.O., 2004. Medium Accuracy INS/GPS Integration in Various GPS Environments. A Thesis for the Degree of Master of Science. Calgary.

Shin, E.-H., 2005. Estimation Techniques for Low Cost Inertial Navigation. A Thesis for the Degree Doctor of Philosophy. Calgary.

Zang, Z., 2003. Integration of GPS with A Medium Accuracy IMU for Metre-Level Positioning. A Thesis for the Degree of Master of Science. Calgary.

Revised April 2017 